\title{
Does gravity cause load-bearing bridges in colloidal and granular systems?
}

\author{
M. C. Jenkins ${ }^{1,2}$, M. D. Haw ${ }^{3}$, G. C. Barker ${ }^{4}$, W. C. K. Poon ${ }^{1}$ and S. U. Egelhaaf ${ }^{2}$ \\ ${ }^{1}$ SUPA and The School of Physics 83 Astronomy, \\ The University of Edinburgh, Kings Buildings, Mayfield Road, \\ Edinburgh EH9 3JZ, UK. ${ }^{2}$ Condensed Matter Physics Laboratory, \\ Heinrich-Heine-University, Universitätsstraße 1, 40225, Düsseldorf, \\ Germany. ${ }^{3}$ Department of Chemical and Process Engineering, \\ University of Strathclyde, James Weir Building, 75 Montrose Street, \\ Glasgow, G1 1XJ, UK. ${ }^{4}$ Institute of Food Research, \\ Norwich Research Park, Colney, Norwich, NR4 7UA, UK.
}

(Dated: January 18, 2011)

\begin{abstract}
We study the load-bearing properties of particulate packings. To investigate the relationship between load-bearing structures ('bridges') and gravity, we experimentally determine bridge statistics in colloidal packings. We vary the effective magnitude and direction of gravity, volume fraction, and interactions, and find that the bridge size distributions depend only on the mean number of neighbors. We identify a universal distribution, in agreement with simulation results for granulars, suggesting that applied loads merely exploit pre-existing bridges, which are inherent structures in dense packings.
\end{abstract}

PACS numbers: $82.70 . \mathrm{D}, 45.70 . \mathrm{Cc}$

Particulate systems are ubiquitous in nature and important in diverse industrial applications. The loadbearing properties of concentrated particulates and their stability under gravity or other external forces are key issues for soils, powders, ceramics, colloids, porous media, packed bed reactors and many other applications. Load-bearing properties have been linked to structural 'bridges', a concept developed in the analysis of computer simulations of granulars [1-8]. Particles belonging to a given bridge mutually stabilize each other against an applied force, such as gravity. Bridges can have various architectures, ranging from linear $[4,6,7]$ to more complex, branched structures $[4,7,8]$. While the bridge size distributions, $P(m)$, putatively link the particle configuration and the load-bearing properties of a packing, aspects of the nature of this relationship remain obscure.

Load-bearing structures are necessary in granular materials to provide stability against gravity. In contrast, thermal energy governs colloidal systems, ensuring that particles remain homogeneously dispersed even if their density differs from the surrounding fluid medium: loadbearing structures are not required for stability against gravity. However, the balance between gravity and thermal agitation can be tuned in colloidal systems. The effect of gravity is quantified by the dimensionless gravitational Péclet number Pe, which compares a single particle's gravitational energy on its own length scale to its thermal energy. In this work we consider equal-sized spheres (radius $R$ ), and define $\mathrm{Pe}=m g R / k_{B} T$, where $m$ is the buoyant mass of a single particle, $g$ the gravitational acceleration and $k_{B} T$ the thermal energy. All particulate systems lie between the colloidal $(\mathrm{Pe} \ll 1)$ and granular $(\mathrm{Pe} \gg 1)$ limits.

The question of how a particle packing can be mechanically stable goes back at least to J. C. Maxwell, who argued that for mechanical equilibrium a particle requires $d+1$ neighbors in $d$ dimensions, but that stability of an assembly of (frictionless) particles requires on average at least $2 d$ neighbors [9-11]. Experimentally, stable sphere packings occur for volume fractions $\phi$ ranging from the so-called random loose packed (RLP) to the random close packed (RCP) states. RCP is the densest non-crystalline state for spheres, and although an exact definition is lacking [12], experiments find $\phi_{\mathrm{RCP}} \simeq 0.64$ [13]. RLP is less well established, but recent experiments suggest $\phi_{\mathrm{RLP}} \simeq 0.55$ [14]. Conceptually the limit of stability is when all particles are stabilized by a single configuration of contacting neighbors $[15,16]$. This state is fragile and sensitive to non-compatible loads [17], which renders reproducible experimentation (and thus comparison of different systems or conditions) difficult.

An outstanding issue is the extent to which concentrated colloidal suspensions are similar to granular materials, i.e. whether the load-bearing properties of particulates for all Pe can be understood within a single framework. Granular packings are stable at volume fractions $\phi_{\mathrm{RLP}} \leq \phi \leq \phi_{\mathrm{RCP}}$, while colloidal suspensions exist for arbitrarily small $\phi$. However, colloids often 'jam' (develop the capacity to resist externally applied forces) around $\phi \simeq 0.55$ [18]. Additionally, how RLP, RCP, and jamming relate to the glass transition in colloids [19], in which all long-range motion ceases on experimental time scales for $\phi \geq \phi_{g}$ ( $\gtrsim 0.58$ for hard spheres) is not clear. It has been suggested that the colloidal glass transition does not occur under reduced gravity [20,21], implying that glasses occur in systems intermediate between colloidal and granular $(\mathrm{Pe} \sim 1)$. Kegel summarized this in stating: 'The important question that remains is if packings can be identified that are present under normal gravity but not under low gravity and vice versa' [21]. In other words, do different packings exist at different Pe?

In this Letter, we contribute towards resolving this is- 

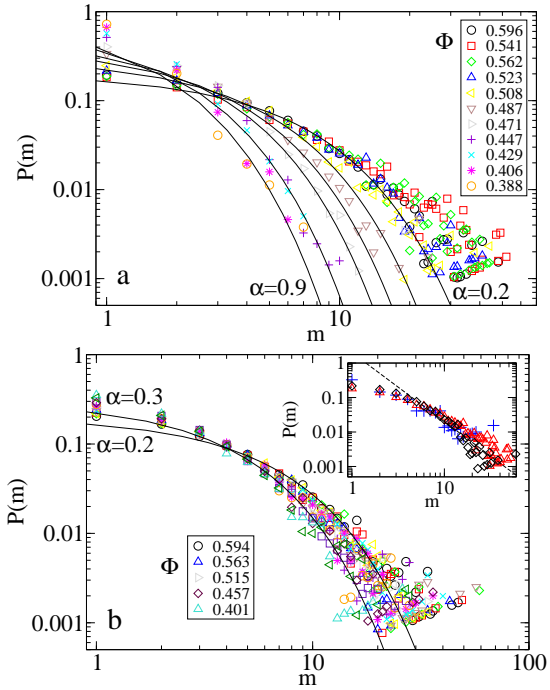

FIG. 1: Bridge size distribution $P(m)$ for $0.39<\phi<0.60$ for the density-matched slightly soft spheres (a) and the nondensity-matched hard-spheres (b). (Only selected datasets labeled for clarity.) The lines represent $P(m)=\alpha \exp (-\alpha m)$ for $\alpha=\{0.2,0.3,0.4,0.5,0.7,0.9\}$ in (a) and $\alpha=\{0.2,0.3\}$ in (b). Inset to b): $P(m)$ for the density-matched $(\triangle)$ and the non-density-matched $(\diamond)$ samples, as well as a simulated granular material $(+)$. All of these samples had $\phi \simeq 0.57$. The dashed line is $P(m)=1.8 m^{-2}$.

sue. We studied hard-sphere colloidal suspensions across a range of low to moderate $\mathrm{Pe}\left(\mathrm{Pe} \approx 10^{-3}\right.$ and $\left.\mathrm{Pe} \approx 4\right)$, which was varied via the degree of density matching. Confocal microscopy was used to record particle coordinates to high accuracy [22], from which, based on methods from granular simulations [4-8], we identified bridges [23] and determined bridge size distributions. We compared these with results from granular simulations $\mathrm{Pe} \gg 1$. This reveals how load-bearing properties are determined by external load. We also examine the role of particle interactions and particle concentration. We find that the concentrated systems studied feature a universal bridge size distribution independent of Pe, and thus gravity, as well as particle interactions, provided mean co-ordination number $\langle z\rangle$ rather than $\phi$ is used as the control parameter. Our results indicate that bridge structures are inherent to particulate systems, irrespective of whether a load is applied, and are thus not load induced but exploited by loads. Bridges thus characterize a particulate system's ability to support a load.

We used sterically-stabilized poly-methylmethacrylate particles (diameter $2 R=2.15 \mu \mathrm{m}$ ) which were fluorescently labeled with 7-nitrobenzo-2-oxa-1,3-diazole. In cis-decalin, the particles behave as nearly hard spheres, crystallizing at $\phi \simeq 0.50$. The density mismatch with the solvent results in a system intermediate between colloids and granulars $(\mathrm{Pe} \simeq 4)$. The second solvent is a nearlydensity-matching mixture of cis-decalin and cycloheptylbromide ( $\simeq 74: 26$ by volume). Centrifugation for several

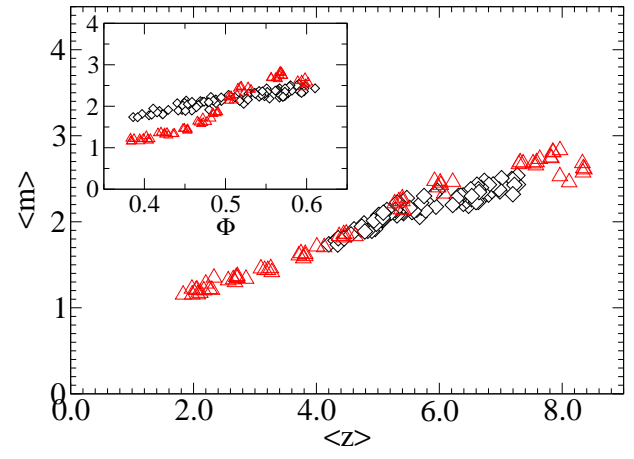

FIG. 2: Mean bridge size $\langle m\rangle$ as a function of mean coordination number $\langle z\rangle$ (and in the inset, of volume fraction $\phi)$ for the density-matched soft spheres $(\triangle)$ and non-densitymatched hard spheres $(\diamond)$.

days at $10^{3} \mathrm{~g}$ caused no observable sedimentation, so that $\mathrm{Pe} \simeq 10^{-3}$; this system is colloidal. The solvent mixture also nearly matches the particles' refractive index, swells the particles slightly, and induces a small charge [24], which was screened by $1.2 \mathrm{mg} / \mathrm{ml}$ of tetrabutylammonium chloride (Fluka), giving a Debye length $\lesssim 100 \mathrm{~nm}$ [24] and conferring a degree of softness to the interparticle potential: these particles crystallized at $\phi \simeq 0.41$, lower than for hard spheres $\left(\phi_{f}=0.494\right)$.

We imaged the particles typically shortly after preparation using a fast confocal scanhead (Visitech VTEye) and a Nikon TE300 inverted microscope, capturing $70 \mu \mathrm{m} \times 70 \mu \mathrm{m} \times 20 \mu \mathrm{m}$ in around $3 \mathrm{~s}$. Particle coordinates were obtained using published procedures [22]. The mean Voronoï volume per particle and the known particle radius allowed us to determine $\phi$.

From the particle coordinates, bridges were identified using a procedure originating from the simulation of granular matter [4-7] and recently adapted for experimental data from colloidal samples [23]. Briefly, bridges are defined as clusters of mutually stabilizing particles, where 'stable' means particles do not move due to gravity, and 'mutual' refers to the fact that bridge members rely on each other for their stability; mutual or cooperative stabilisations are necessary to explain stable piles of various packing fraction [23]. During bridge finding, neighbors were considered contacting if their centers were within $2 c R$ with the cutoff value $c=1.12$, which allowed for experimental uncertainties in the particle location and polydispersity. Furthermore, the single stabilizing subset for each stable particle was chosen based on a 'lowest mean-squared separation' (LSQS) criterion, which we have shown to compensate for the experimental uncertainties already mentioned [23]. From the identified contacting neighbors for each particle, we also calculated the mean co-ordination number, $\langle z\rangle$.

Of primary interest is the bridge size distribution, i.e., the probability that a particle belongs to a bridge of size $m, P(m)=m N(m) / N_{\text {tot }}$ with $N(m)$ the number of bridges with $m$ members and $N_{\text {tot }}$ the total number 


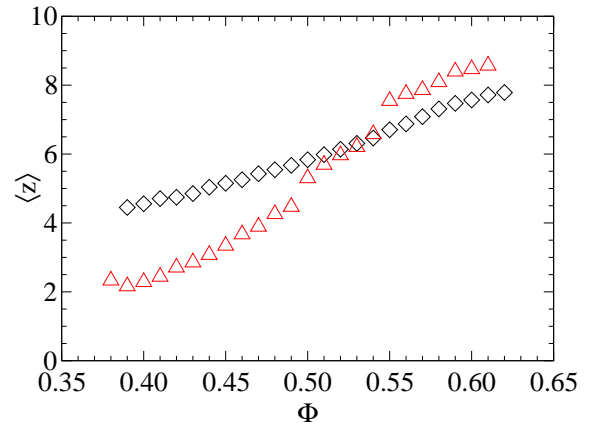

FIG. 3: Mean co-ordination number $\langle z\rangle$ as a function of volume fraction $\phi$ for the density-matched soft $(\triangle)$ and nondensity-matched hard spheres $(\diamond)$.

of stable particles. The $P(m)$ obtained for various $\phi$ for the slightly soft, low Pe and the hard, moderate Pe systems are given in Fig. 1. For a given moderate $\phi$, the $P(m)$ for the two systems differ significantly, while for high $\phi$ samples, they are quantitatively identical within experimental uncertainty, Fig. 1.

In the slightly soft, low Pe system, Fig. 1a, $P(m)$ noticeably shifts upwards with $\phi$ until $\phi \gtrsim 0.54$, which is also reflected in an increase in the mean bridge size, $\langle m\rangle$ (Fig. 2, inset). Thereafter, $P(m)$ changes little, suggesting that an underlying distribution $P_{0}(m)$ is achieved for sufficiently high $\phi$. For the hard, moderate Pe system, $P(m)$ hardly changes in the range $0.39 \leq \phi \leq 0.60$, Fig. 1b, although there is a slight systematic increase in $\langle m\rangle$ with $\phi$ (Fig. 2, inset).

To compare the measured $P(m)$ with a system in the limit $\mathrm{Pe} \gg 1$, we simulated a granular packing following published procedures [6]. Briefly, 2200 spheres $(2 R=1)$ in a (periodic) box with a $6 \times 6$ square base were allowed to form a deposit with $\phi \simeq 0.57$ and mean co-ordination number $\langle z\rangle \simeq 4.7$ using a 'drop-and-roll' procedure designed to capture mutual stabilizations. Significantly, we obtained the same $P(m)$ to within errors as the limiting $P_{0}(m)$ found in both experimental systems, Fig. 1b, inset. Differences at the highest $m$ are artefacts [28]. These observations suggest an underlying general bridge size distribution, $P_{0}(m)$, in densely packed soft and hard spheres at all Pe, i.e. independent of the effect of gravity and the system.

We compare the forms of our $P(m)$ with those found in simulations. Simple arguments suggest that at low $\mathrm{m}$, linear bridges dominate with $P(m)=\alpha e^{-\alpha m}$, with $p=e^{-\alpha}$ the probability that an existing linear bridge is extended from size $\mathrm{m}$ to $\mathrm{m}+1$ [4]. Above $m \simeq 10$, linear bridges become unlikely and randomly-branched bridges with $P(m) \propto m^{-\tau}$ are observed. The $P(m)$ are well described by $P(m)=\alpha e^{-\alpha m}$ over the whole range of $\phi$, albeit less well for large $m$, Figs. 1, 4 (inset). For $m \gtrsim 10$, the data are also consistent with $P(m) \propto m^{-\tau}$ with $\tau \simeq 2$ (Fig. 1). Thus, small bridges $(m \lesssim 10)$ are linear, while larger bridges (found only at large $\phi$ ) might

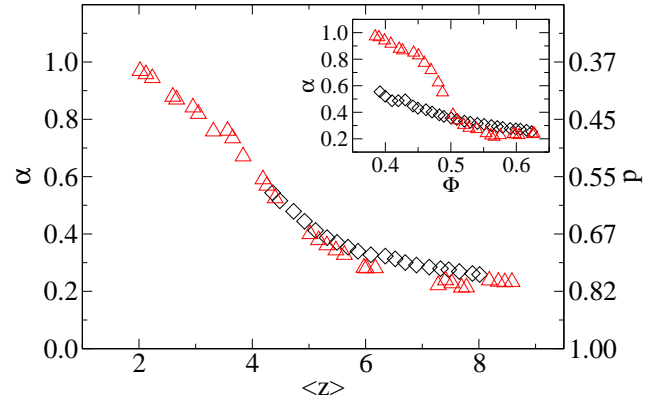

FIG. 4: Exponent $\alpha$ in the fit $P(m)=\alpha e^{-\alpha m}$ as a function of $\langle z\rangle$ (and, in the inset, of $\phi$ ) for the non-density-matched hard- $(\diamond)$ and density-matched slightly soft-spheres $(\triangle)$. The right-hand axis shows the corresponding probability $p=e^{-\alpha}$.

be branched, in agreement with simulations $[4,7]$.

$\langle m\rangle, \alpha$, and the concentration above which $P(m)$ approaches $P_{0}(m)$ are Pe- and interaction-dependent. However, the quite different $\phi$-dependences of $\langle m\rangle$ and $\alpha$ for the two systems collapse onto a single curve when plotted vs. $\langle z\rangle$, Figs. 2, 4. This reveals that there is a universal bridge structure distribution in these particulates, independent of $\mathrm{Pe}$ and interaction, when measured as a function of $\langle z\rangle$. It is reasonable that $\langle z\rangle$ rather than $\phi$ controls bridge statistics, since $\phi$ measures space filling while $\langle z\rangle$ reflects connectivity, which is necessary for load-bearing ability. The function $\alpha(\langle z\rangle)$ appears to asymptote to a constant value at large $\langle z\rangle$, consistent with the approach to the limiting distribution $P_{0}(m)$.

Having investigated the effect of the magnitude of the gravitational force on $P(m)$, we consider the sensitivity of $P(m)$ to the direction of the applied force. $P(m)$ was determined for a fully-sedimented non-density-matched sample with $\phi \approx 0.647$, Fig. $5(\bullet)$. The coordinates were then rotated electronically through $90^{\circ}(\boldsymbol{\square})$, as well as inverted $(\downarrow)$, and the analysis repeated, giving $P(m)$ perpendicular and opposite to the applied force, Fig. 5. The $P(m)$ are identical to within experimental error [29], suggesting that potentially load-bearing structures, i.e. bridges, are present independent of applied load. This is consistent with bridge structures also being found in dilute samples, where there is no 'stability' against gravity and at low Pe where there is no significant gravitational load. We conclude that bridge structures are formed without applied load and are only exploited for stability once a load is applied, and that bridge structures with the potential to support a load are inherent to particulate systems across the whole range of Pe.

Lastly, we investigate the effect of crystallinity on $P(m)$ for samples with the same $\langle z\rangle$. We selected some partially crystalline $\mathrm{Pe} \simeq 10^{-3}$ samples, as determined by the rotationally-invariant global orientational order parameter $Q_{6}$ [25-27], which we consider relative to the value in a perfect face-centered cubic crystal, $Q_{6}^{\mathrm{FCC}} \simeq 0.57452$. We determined $P(m)$ for amorphous 


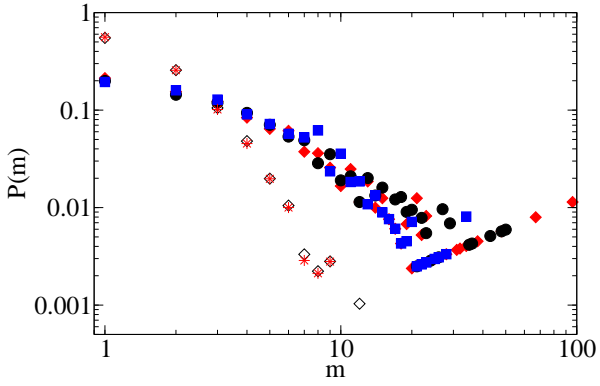

FIG. 5: Bridge size distribution $P(m)$ for density-matched amorphous $\left(Q_{6} / Q_{6}^{\mathrm{FCC}}<0.05, \diamond\right)$ and (partially) crystalline $\left(Q_{6} / Q_{6}^{\mathrm{FCC}}>0.70,{ }^{*}\right)$ samples with similar $\langle z\rangle(2.877 \pm 0.054$ and $2.873 \pm 0.032$ respectively), and for a non-density-matched sample, $\phi \approx 0.647$, obtained in its natural orientation $(\bullet)$, electronically rotated by $90^{\circ}(\mathbf{\square})$, and upside down, i.e. $z$ co-ordinates artificially inverted $(\diamond)$.

$\left(Q_{6} / Q_{6}^{\mathrm{FCC}}<0.05\right)$ and crystalline $\left(Q_{6} / Q_{6}^{\mathrm{FCC}}>0.70\right)$ samples of similar $\langle z\rangle \approx 2.9$, Fig. 5. The two $P(m)$ are remarkably similar, indicating that the precise spatial configuration of neighbors is not crucial as long as $\langle z\rangle$ is similar; this recommends closer investigation of the importance of the angular distribution of neighbors.

In conclusion, we have studied the bridge size distribution, $P(m)$, in two model colloidal suspensions: hard spheres at $\mathrm{Pe} \simeq 4$ and slightly soft spheres at $\mathrm{Pe} \simeq 10^{-3}$. The bridge statistics scale with the mean co-ordination number, $\langle z\rangle$, rather than the volume fraction, $\phi$. A generic bridge size distribution, $P_{0}(m)$, is achieved for $\langle z\rangle \gtrsim 5$.0, which also agreed with $P(m)$ for simulated granular materials, and for different orientations of the applied force. Moreover, amorphous and partially crystalline samples with the same $\langle z\rangle$ also showed the same measured $P(m)$. These observations are consistent with the fact that the co-ordination number $\langle z\rangle$ reflects 'connectivity', which is more important than space-filling, i.e. the volume fraction $\phi$. Connectedness, necessary for one particle to transmit a force to another, depends not only on the volume fraction $\phi$, but also on the particle interactions. Remarkably, this applies not only to the limiting distribution $P_{0}(m)$, but also to the bridge size distributions for dilute samples with markedly fewer contacting neighbors, and density-matched samples where a load is essentially absent. These 'bridges' can be neither load-bearing nor load-induced. Inherent structures with the potential to bear loads are formed without gravity and then exploited by applied forces to provide stability. Bridges thus characterize the load-bearing ability, not the actual degree of load-bearing, of a packing. More information might be extracted from the details of the individual stabilizations which lead to bridges. To answer Kegel's question [21], we do not find different structures in packings with different degrees of applied load: the same universal distribution of bridge structures is present regardless of applied load.

We thank A. Schofield for supplying the particles. This work was funded by the EPSRC and Rhodia-Centre de Recherches et Technologies d'Aubervilliers.
[1] G. Y. Onoda and E. G. Liniger, Phys. Rev. Lett. 64, 2727 (1990).

[2] G. T. Nolan and P. E. Kavanagh, Powder Technol. 72, 149 (1992).

[3] R. Arévalo, D. Maza, and L. A. Pugnaloni, Phys. Rev. E 74, 021303 (2006)

[4] A. Mehta, Granular Physics (CUP, 2007).

[5] L. A. Pugnaloni and G. C. Barker, Physica A 337, 428 (2004).

[6] L. A. Pugnaloni, G. C. Barker, and A. Mehta, Adv. Complex Systems 4, 289 (2001).

[7] A. Mehta, G. C. Barker, and J. M. Luck, J. Stat. Mech. Theory E P10014 (2004).

[8] A. Mehta, G. C. Barker, and J.-M. Luck, Phys. Today 62, 40 (2009).

[9] J. C. Maxwell, Philos. Mag. 27, 294 (1864).

[10] S. Alexander, Phys. Rep. 296, 65 (1998).

[11] M. Wyart, Ann. Phys.-Paris 30, 1 (2005).

[12] S. Torquato, T. M. Truskett, and P. G. Debenedetti, Phys. Rev. Lett. 84, 2064 (2000).

[13] T. Aste and D. L. Weaire, The Pursuit of Perfect Packing (Institute of Physics, 2000).

[14] M. Jerkins et al., Phys. Rev. Lett. 101, 018301 (2008).

[15] R. Blumenfeld, Phys. Rev. Lett. 93, 108301 (2004).

[16] R. Blumenfeld, S. F. Edwards, and R. C. Ball, J. Phys.: Condens. Matter 17, S2481 (2005).
[17] M. E. Cates, J. P. Wittmer, J.-P. Bouchaud, and P. Claudin, Phys. Rev. Lett. 81, 1841 (1998).

[18] M. D. Haw, Phys. Rev. Lett. 92, 185506 (2004).

[19] P. N. Pusey and W. van Megan, Phys. Rev. Lett. 59, 2083 (1987).

[20] J. Zhu et al., Nature 387, 883 (1997).

[21] W. K. Kegel, Langmuir 16, 939 (2000).

[22] M. C. Jenkins and S. U. Egelhaaf, Adv. Colloid Interface Sci. 136, 65 (2008).

[23] M. C. Jenkins et al., Soft Matter 7, (probably) (2011).

[24] A. Yethiraj and A. van Blaaderen, Nature 421, 513 (2003).

[25] P. J. Steinhardt, D. R. Nelson, and M. Ronchetti, Phys. Rev. B 28, 784 (1983).

[26] J. S. van Duijneveldt and D. Frenkel, J. Chem. Phys. 96, 4655 (1992).

[27] P. R. ten Wolde, M. J. Ruiz-Montero, and D. Frenkel, J. Chem. Phys. 104, 9932 (1996).

[28] The straight portions of $P(m)$ at large $m$ (Figs. 1, 5) are artefacts of poor statistics; at large $m, N(m)$ is typically one, so $P(m)=m N(m) / N_{\text {tot }}$, is a line of slope unity on a log-log plot.

[29] The volume analyzed, $\approx 75 \mu \mathrm{m} \times 75 \mu \mathrm{m} \times 12 \mu \mathrm{m}$, was not cubic. The analysis repeated for an isotropic $(12 \mu \mathrm{m})^{3}$ sub-volume yielded noisier but comparable $P(m)$. 